\title{
Amplitude Response Curves of Frequency-Locked Rotations
}

\author{
Yoshihiko SUSUKI ${ }^{\dagger \mathrm{a})}$, Member and Yoshisuke UEDA ${ }^{\dagger \dagger * \mathrm{~b})}$, Fellow
}

\begin{abstract}
SUMMARY This letter studies frequency-locked rotations in a phaselocked loop (PLL) circuit as FM demodulator. A rotation represents a desynchronized steady state in the PLL circuit and is regarded as another type of self-excited oscillations with natural rotation frequencies. The rotation frequency can be locked at driving frequencies of modulation signals. This letter shows response curves for harmonic amplitude of frequencylocked rotations. They have several different features from response curves of van der Pol oscillator.

key words: phase-locked loop, frequency entrainment, rotation, response curve
\end{abstract}

\section{Introduction}

Phase-locked loop (PLL) is well known as an important technique in communication engineering [1], [2]. PLL consists of phase comparator, loop filter, and voltage-controlled oscillator. Nonlinear oscillations in PLL circuit have been studied in [3]-[5]. Their understanding is important for the application of PLL to various electrical and electronic circuits.

A dynamical system that is analyzed in [3]-[5] to reveal the dynamics of a PLL circuit has two steady states. One is a stable equilibrium point which corresponds to the phase-locked steady state. The other is a stable limit cycle resulting from the periodic nature of phase space, called stable limit cycle of the second kind [6] or stable rotation [7]. The rotation represents a desynchronized steady state in the PLL circuit and is regarded as another type of self-excited oscillations with natural rotation frequencies. Such rotating steady states in PLL are studied to analyze its pull-in range using the Galerkin method [3], and those in Josephson junction are also examined to clarify its current-voltage characteristics [7].

This letter considers frequency-locked rotations in a PLL circuit as FM demodulator. Frequency entrainment or locking by external force is well known in periodically driven self-oscillatory systems [6], [8], [9]. In the van der Pol oscillator [8], we focus on stable limit cycles of the first

Manuscript received December 1, 2006.

Manuscript revised February 13, 2007.

Final manuscript received May 9, 2007.

${ }^{\dagger}$ The author is with Department of Electrical Engineering, Kyoto University, Kyoto-shi, 615-8510 Japan.

${ }^{\dagger \dagger}$ The author is with Department of Complex Systems, Future University-Hakodate, Hakodate-shi, 041-8655 Japan.

*Presently, with Waseda University.

a)E-mail: susuki@ dove.kuee.kyoto-u.ac.jp

b)E-mail: ueda.yoshi@gmail.com

DOI: 10.1093/ietfec/e90-a.10.2250 kind [6] or stable librations [7], and we examine locked librations at driving frequencies of external force to the oscillator. Amplitude response curves in [6], [8], [9] are derived to show characteristic features of frequency-locked librations. In the case of the PLL circuit, the rotation frequency can be also locked at driving frequencies of modulation signals. The corresponding solution to the locked state is discussed in Josephson Junction circuit [10]-[12]. The frequency-locked rotations are of significance for the analysis of pull-in range under modulation signals. Unfortunately, they have not been fully understood in the contexts of nonlinear oscillation and PLL, and the difference in frequency-locked librations and rotations has not been also examined. This letter shows amplitude response curves of frequency-locked rotations in the PLL circuit. The obtained curves imply that they have different features from the response curves of van der Pol oscillator.

\section{Mathematical Model}

This section introduces a mathematical model which describes the dynamics of a PLL circuit [4]. The PLL circuit includes a sinusoidal phase comparator, a lag-lead loop filter, and a voltage-controlled oscillator. Suppose that $\phi$ denotes the phase error of input signals to the phase comparator, then the phase error dynamics are represented in [4] by the following system of differential equations:

$$
\left\{\begin{aligned}
\frac{\mathrm{d} \phi}{\mathrm{d} t}=y, & \\
\frac{\mathrm{d} y}{\mathrm{~d} t}= & -\beta y+\beta \sigma-\sin \phi \\
& +m \sqrt{\beta^{2}+\Omega^{2}} \cos \Omega t .
\end{aligned}\right.
$$

$\beta$ and $\sigma$ are the fixed parameters, and $m$ and $\Omega$ are the parameters of modulation signal. The parameters are in per unit system. The driving term $m \sqrt{\beta^{2}+\Omega^{2}} \cos \Omega t$ is slightly modified from the original one in [4] for simplicity of the present analysis. The two variables $(\phi, y)$ in the system (1) belong to cylindrical phase plane because of the periodic restoring term. The periodic nature of phase space results in the occurrence of stable rotations. Additionally, to derive theoretical response curves in Sect. 3, a smooth function $S(\phi, y)$ for the system (1) is defined by

$$
S(\phi, y)=\frac{1}{2} y^{2}-\cos \phi-\beta \sigma \phi .
$$

The time derivative of $S$ for any solution $(\phi(t), y(t))$ is now 
given by

$$
\begin{aligned}
\frac{\mathrm{d} S}{\mathrm{~d} t}(\phi(t), y(t))= & -\beta\{y(t)\}^{2} \\
& +y(t) m \sqrt{\beta^{2}+\Omega^{2}} \cos \Omega t .
\end{aligned}
$$

By integrating the above equality from $t=0$ to $\tau$, the following equality is derived:

$$
\begin{gathered}
S(\phi(\tau), y(\tau))-S(\phi(0), y(0))=-\int_{0}^{\tau} \beta\{y(t)\}^{2} \mathrm{~d} t \\
+\int_{0}^{\tau} y(t) m \sqrt{\beta^{2}+\Omega^{2}} \cos \Omega t \mathrm{~d} t .
\end{gathered}
$$

The equality (4) is used for the derivation of response curves in Sect. 3.

\section{Amplitude Response Curves}

This letter shows amplitude response curves of frequencylocked rotations against the parameters $\Omega$ and $m$. The system (1) under $m=0$ and $|\beta \sigma|>4 \beta / \pi$ has one stable rotation with angular frequency $\Omega_{0}$. The condition of $\beta$ and $\sigma$ is obtained via the Melnikov's method [13]. The corresponding solution $\phi_{0}(t)$ to the stable rotation is represented using periodic function $x_{0}(t)$ by

$$
\phi_{0}(t)=\Omega_{0} t+x_{0}(t), \quad x_{0}(t) \equiv x_{0}\left(t+\frac{2 \pi}{\Omega_{0}}\right) .
$$

Figure 1 shows the waveform of stable rotation in the system (1) under $m=0, \beta=0.56$, and $\sigma=1.7$. The setting of $\beta$ is based on [4], [5]. $\Omega_{0}$ is 1.6 under the parameter setting. Within the small difference between natural rotation frequency $\Omega_{0}$ and driving frequency $\Omega$, it is expected that we observe a locked rotation at the driving frequency $\Omega$. The type of frequency entrainment is called harmonic entrainment.

Here, the averaging method [9], [14] is used for the
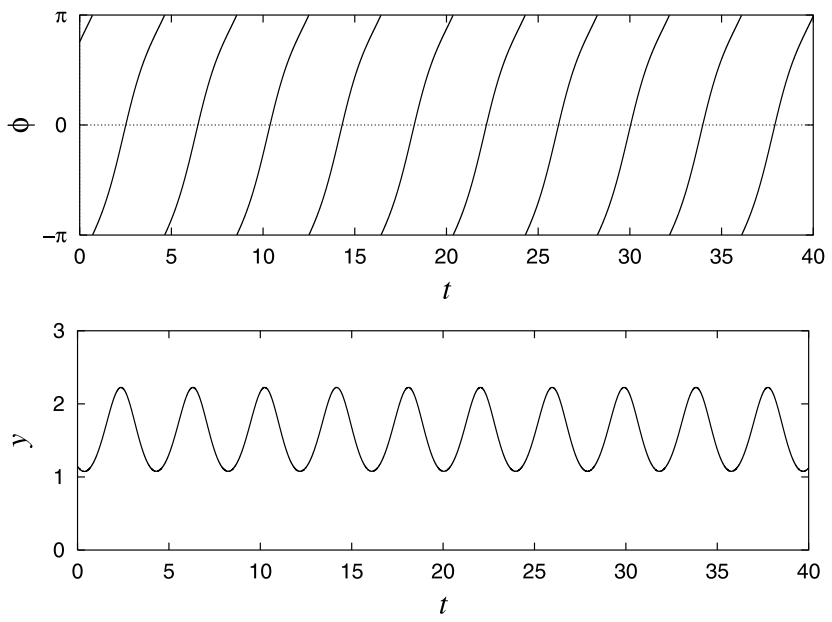

Fig. 1 Waveform of stable rotation in the system (1) under $m=0, \beta=$ 0.56 , and $\sigma=1.7$ derivation of response curves. At the harmonic entrained states, the corresponding solution $\phi(t)$ can be represented using periodic function $x(t)$ as follows:

$$
\phi(t)=\Omega t+x(t), x(t) \equiv x\left(t+\frac{2 \pi}{\Omega}\right) .
$$

It is now assumed that the solution $x(t)$ is approximated as follows:

$$
x(t)=\frac{A_{0}}{2}+A_{1}(t) \cos \left\{\Omega t+\varphi_{1}(t)\right\},
$$

where $A_{0}$ is constant. $A_{1}$ and $\varphi_{1}$ are assumed to be slowly varying functions of $t$ and are constant at the entrained states. By substituting $\phi(t)=\Omega t+A_{0} / 2+A_{1}(t) \cos \left\{\Omega t+\varphi_{1}(t)\right\}$ and $y(t)=\Omega-\Omega A_{1}(t) \sin \left\{\Omega t+\varphi_{1}(t)\right\}$ in the system $(1)$ and eliminating all oscillatory terms containing $\cos n \Omega t$ and $\sin n \Omega t$ for $n=1,2, \ldots$, the following system is obtained:

$$
\left\{\begin{aligned}
\frac{\mathrm{d} A_{1}}{\mathrm{~d} t}= & \frac{1}{2 \Omega}\left[\left\{J_{0}\left(A_{1}\right)+J_{2}\left(A_{1}\right)\right\} \cos \left(\frac{A_{0}}{2}-\varphi_{1}\right)\right. \\
& \left.-m \sqrt{\beta^{2}+\Omega^{2}} \sin \varphi_{1}-\beta \Omega A_{1}\right], \\
A_{1} \frac{\mathrm{d} \varphi_{1}}{\mathrm{~d} t}= & \frac{1}{2 \Omega}\left[\left\{J_{0}\left(A_{1}\right)-J_{2}\left(A_{1}\right)\right\} \sin \left(\frac{A_{0}}{2}-\varphi_{1}\right)\right. \\
& \left.-m \sqrt{\beta^{2}+\Omega^{2}} \cos \varphi_{1}-\Omega^{2} A_{1}\right],
\end{aligned}\right.
$$

where $J_{n}$ for $n=0,2$ are the Bessel functions of the first kind. The system (8) approximately represents the dynamics of $A_{1}$ and $\varphi_{1}$ under the above assumption and is regarded as a first-order averaged system.

Theoretical response curves of frequency-locked rotations are derived through the above preliminary. The three variables $A_{0}, A_{1}, \varphi_{1}$ are here required for the derivation. In other words, we need to obtain independent three determining equations. Two of the required equations are induced by equating the right-hand side of the averaged system (8) to zero. The remaining equation is obtained from the equality (4). $A_{1}$ and $\varphi_{1}$ are constant at the entrained states. Then, by substituting the above $\phi(t)$ and $y(t)$ in the equality (4) with $\tau=2 \pi / \Omega$, the following third equation for the derivation of response curves is derived:

$$
-2 \pi \beta \sigma=-\pi \beta \Omega\left(2+A_{1}^{2}\right)-\pi m \sqrt{\beta^{2}+\Omega^{2}} A_{1} \sin \varphi_{1} .
$$

Unfortunately, the three dimensional determining equations have not any explicit solution. The solutions are numerically obtained with the Newton-Raphson method in the next paragraph.

Figure 2 shows several response curves of frequencylocked rotations under $\beta=0.56$ and $\sigma=1.7$. Figure 2(a), which shows the harmonic amplitude $A_{1}$, is obtained by plotting the above three equations in $\Omega-A_{1}$ plane for several values of $m$. Figure 2(b), which describes the harmonic amplitude of $x(t)$, is given with numerical integration of the original system (1). The response curve at $m=0.6$ in Fig. 2(a) is here described in the absolute value. Each closed 


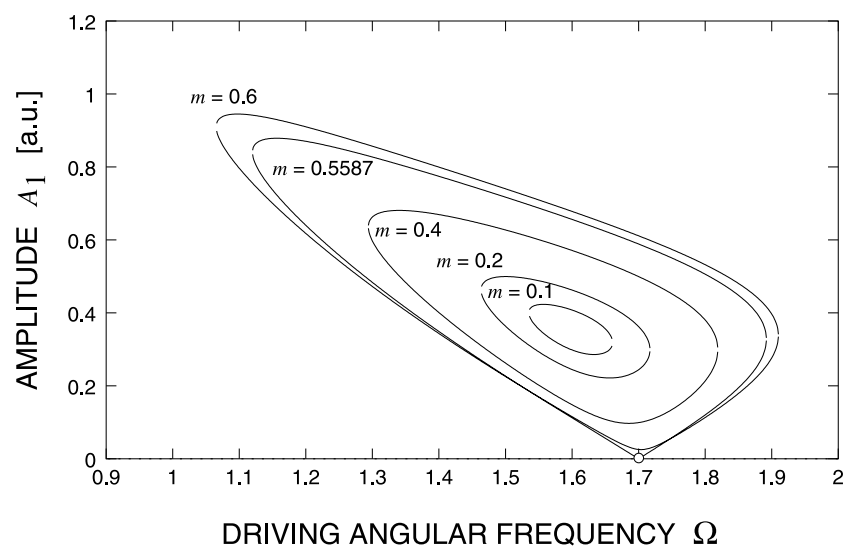

(a) Theoretical result with averaging method.

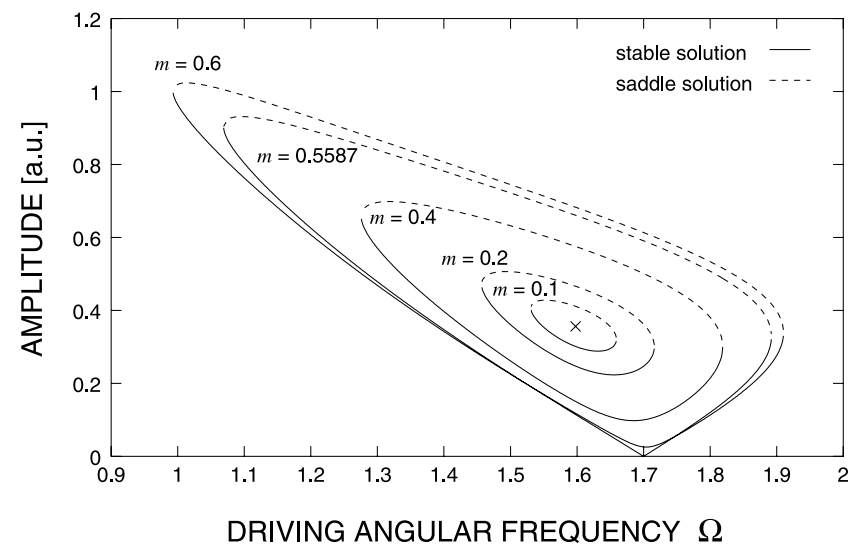

(b) Numerical result with numerical integration.

Fig. 2 Amplitude response curves of frequency-locked rotations under $\beta=0.56$ and $\sigma=1.7$.

curve in Fig. 2(a) contains amplitude response of both stable and unstable periodic solutions. On the other hand, in Fig. 2(b), the solid lines describe the response curves for stable solutions, and the broken ones for saddle-type solutions. The symbol $\times$ in Fig. 2(b) denotes the parameter set $(\Omega, m)=\left(\Omega_{0}, 0\right)$. The corresponding value of $A_{1}$ is equal to the harmonic amplitude of $x_{0}(t)$ of the stable rotation in Fig. 1. Additionally, Fig. 2(b) suggests that the harmonic amplitude of $x(t)$ is precisely zero at the parameter set $(\Omega, m)=(1.7,0.5587)^{\dagger}$. It should be also noted at the parameter set that all the harmonics of $x(t)$ have zero amplitude in Fig. 2(a). Figure 2 implies under the parameter setting that the theoretical result with averaging method almost corresponds to that with numerical integration.

\section{Discussion}

This letter showed amplitude response curves of frequencylocked rotations in the PLL circuit. Figure 2 provides a characteristic feature of the frequency-locked rotations. The obtained response curves are here considered by comparison

${ }^{\dagger}$ The corresponding point in Fig. 2(a), denoted by the white circle, is not uniquely defined because of the lack of determining equations. with frequency-locked librations. The response curves in van der Pol oscillator are adopted for the comparison, which are given in Fig. 2 of [8], Fig. 113.1 of [6], or Fig. 12.1 of [9]. The shape of response curves in Fig. 2 differs from that of van der Pol oscillator. In the van der Pol oscillator, stable solutions have the maximum amplitude near the natural frequency. On the other hand, in the PLL circuit, every harmonic amplitude for stable solutions attains the minimum value near the natural rotation frequency $\Omega_{0}$. Furthermore, it gradually increases to each side of the minimum value in a different manner from the response curves in van der Pol oscillator. The obtained response curves hence delineate some different features in the frequency-locked rotations and librations. They lead to the detailed analysis of frequencylocked rotations and librations [15].

\section{Acknowledgments}

The first author appreciates Professor Takashi Hikihara for his fruitful suggestions and Mr. Yuuichi Yokoi for valuable discussion.

\section{References}

[1] A.J. Viterbi, Principles of Coherent Communication, McGraw-Hill, New York, 1966.

[2] R.E. Best, Phase-Locked Loops: Design, Simulation, and Applications, McGraw-Hill, New York, 1999.

[3] T. Endo and K. Tada, "Analysis of the pull-in range of phase-locked loops by the Galerkin method," IECE Trans. Commun. (Japanese Edition), vol.J68-B, no.2, pp.236-243, Feb. 1985.

[4] T. Endo and L.O. Chua, "Chaos from phase-locked loops," IEEE Trans. Circuits Syst., vol.35, no.8, pp.987-1003, Aug. 1988.

[5] T. Endo, W. Ohno, and Y. Ueda, "Explosion of strange attractors and crisis-induced intermittency from a forced phase-locked loop circuit: Theory and experiments," Int. J. Bifurcat. Chaos, vol.10, no.4, pp.891-912, April 2000.

[6] M. Minorsky, Introduction to Non-Linear Mechanics, Edwards Brothers, Ann Arbor, 1947.

[7] S.H. Strogatz, Nonlinear Dynamics and Chaos, Addison-Wesley, Reading, 1994

[8] B. van der Pol, "Forced oscillations in a circuit with non-linear resistance (Reception with reactive triode)," Philos. Mag., vol.3, pp.6580, 1927.

[9] C. Hayashi, Nonlinear Oscillations in Physical Systems, McGrawHill, New York, 1964.

[10] V.N. Belykh, N.F. Pederson, and O.H. Soerensen, "ShuntedJosephson-junction model. II. The nonautonomous case," Phys. Rev. B, vol.16, no.11, pp.4860-4871, Dec. 1977.

[11] R.L. Kautz, "The ac Josephson effect in hysteric junctions: Range and stability of phase lock," J. Appl. Phys., vol.52, no.5, pp.35283541, May 1981.

[12] D. D'humieres, M.R. Beasley, B.A. Huberman, and A. Libchaber, "Chaotic states and routes to chaos in the forced pendulum," Phys. Rev. A, vol.26, no.6, pp.3483-3496, Nov. 1982.

[13] J. Guckenheimer and P. Holmes, Nonlinear Oscillations, Dynamical Systems, and Bifurcations of Vector Fields, Springer-Verlag, New York, 1983.

[14] J.K. Hale, Ordinary Differential Equations, John Wiley \& Sons, New York, 1969

[15] Y. Susuki, Y. Yokoi, and T. Hikihara, "Energy-based analysis of frequency entrainment described by van der Pol and phase-locked loop equations," CHAOS, vol.17, art.no.023108, June 2007. 